\title{
JULIA'S LEMMA ON THE HYPERBOLIC DISK
}

\author{
Árpád Baricz*, Saminathan Ponnusamy ${ }^{\dagger}$ and Csaba Varga
}

Babeş-Bolyai University, Department of Economics, Cluj-Napoca 400591, Romania and Óbuda University, Institute of Applied Mathematics

1034 Budapest, Hungary; bariczocsi@yahoo.com

Indian Statistical Institute (ISI), Chennai Centre, SETS, MGR Knowledge City

CIT Campus, Taramani, Chennai 600 113, India; samy@isichennai.res.in, samy@iitm.ac.in

Babeş-Bolyai University, Faculty of Mathematics and Computer Science 400084 Cluj-Napoca, Romania; csvarga@cs.ubbcluj.ro

\begin{abstract}
This paper contains an extension of the Julia-Miller-Mocanu lemma for holomorphic functions defined on the unit disk $\mathbf{D}$ endowed by the hyperbolic metric on $\mathbf{D}$. The extension is made by following on the one hand the way of the differential subordinations theory and on the other hand the method of Lagrange multipliers, like in an extension of the Julia-Miller-Mocanu lemma for holomorphic mappings in $\mathbf{C}^{n}$.
\end{abstract}

\section{Introduction and preliminaries}

The classical Julia lemma continues to enjoy the attention of many mathematicians in problems concerning univalent functions. We begin the discussion by recalling it.

Lemma A. Let $\left|z_{0}\right|<1$ and $r_{0}=\left|z_{0}\right|$. Let $f(z)=\sum_{k=n}^{\infty} a_{k} z^{k}$ be continuous on $|z| \leq r_{0}$ and holomorphic on $\left\{z:|z|<r_{0}\right\} \cup\left\{z_{0}\right\}$ with $f(z) \not \equiv 0$ and $n \geq 1$. If $\left|f\left(z_{0}\right)\right|=\max _{|z| \leq r_{0}}|f(z)|$, then $z_{0} f^{\prime}\left(z_{0}\right) / f\left(z_{0}\right)$ is real number and $z_{0} f^{\prime}\left(z_{0}\right) / f\left(z_{0}\right) \geq n$.

Under the hypothesis of Lemma A, Miller and Mocanu [MM1] concluded that

$$
\operatorname{Re}\left(\frac{z_{0} f^{\prime \prime}\left(z_{0}\right)}{f^{\prime}\left(z_{0}\right)}+1\right) \geq m
$$

where $m=z_{0} f^{\prime}\left(z_{0}\right) / f\left(z_{0}\right)$. Lemma A coupled with the last inequality led to the development of the theory of differential subordination (see [MM2] and the monograph of Miller and Mocanu [MM3, p. 19]). Lemma A is often quoted as Jack's lemma or Clunie-Jack's lemma although this fact was known much before the work of Jack ([Bo, Cl] and [Ja, Lemma 1]). See the article of Boas [Bo] for historical commentary and the application of Julia's lemma. We refer to [CV2] for an extension of Julia and Miller-Mocanu's results for holomorphic mappings in $\mathbf{C}^{n}$, see also [CV1, KP] for more details on this topic. Our aim in this article is to extend the Julia lemma on the unit disk $\mathbf{D}:=\{z \in \mathbf{C}:|z|<1\}$ endowed with the hyperbolic metric.

doi:10.5186/aasfm.2015.4054

2010 Mathematics Subject Classification: Primary 32H02, 30F45, 42B15.

Key words: Holomorphic functions, hyperbolic metric, Lagrange multipliers.

* The research of Á. Baricz was supported by a grant supporting excellence in scientific research of the Babeş-Bolyai University, project number GSCE-30246/2015.

$\dagger$ This second author is on leave from the Indian Institute of Technology Madras. 
Recall that the hyperbolic metric on the unit disk $\mathbf{D}$, also called the Poincaré metric on $\mathbf{D}$, is the Riemannian metric $\lambda_{\mathbf{D}}(z)|d z|$, where $\lambda_{\mathbf{D}}(z)=1 /\left(1-|z|^{2}\right)$. Often we work with arc length element

$$
d s=\lambda_{\mathbf{D}}(z)|d z| .
$$

Thus, we define the hyperbolic length of a piecewise $C^{1}$ curve $\gamma$ in $\mathbf{D}$ as $\int_{\gamma} d s$. In particular, the distance function induced by the hyperbolic metric on $\mathbf{D}$ is

$$
\rho(a, b)=\inf \int_{\gamma} d s,
$$

where the infimum is taken over all piecewise $C^{1}$ curves $\gamma$ in $\mathbf{D}$ joining $a$ and $b$. It is to be recalled that the induced distance on $\mathbf{D}$, also called hyperbolic (or Poincaré) distance on $\mathbf{D}$, is given by

$$
\rho(a, b)=\operatorname{arctanh}\left|\frac{b-a}{1-\bar{a} b}\right|, \quad a, b \in \mathbf{D}
$$

and, in particular,

$$
\rho(0, b)=\operatorname{arctanh}|b|=\frac{1}{2} \log \left(\frac{1+|b|}{1-|b|}\right), \quad b \in \mathbf{D} .
$$

Both the hyperbolic distance and the hyperbolic metric on $\mathbf{D}$ are invariant under conformal automorphisms of $\mathbf{D}$. The geodesics of the hyperbolic metric on $\mathbf{D}$ are circular arcs and line segments which are orthogonal to the unit circle.

Now, the classical Schwarz-Pick lemma can be stated in terms of the hyperbolic metric rather than the Euclidean metric and its corollary (see [IT, Os]), which will be used in the sequel.

Lemma B. Every holomorphic mapping $f: \mathbf{D} \rightarrow \mathbf{D}$ satisfies $\left|f^{*}(z)\right| \leq 1$ in $\mathbf{D}$, where $f^{*}(z)$ denotes the hyperbolic derivative of $f$ at $z$ given by

$$
f^{*}(z)=\frac{1-|z|^{2}}{1-|f(z)|^{2}} f^{\prime}(z) \text { for all } z \in \mathbf{D} \text {. }
$$

For a new version of Schwarz-Pick lemma, we refer to the article of Beardon [Be] and for an extension of this result for hyperbolic derivatives one can refer to $[\mathrm{BC}]$. A non-Euclidean version of Lemma $\mathrm{B}$ is the following:

Corollary C. Every holomorphic mapping $f: \mathbf{D} \rightarrow \mathbf{D}$ satisfies

$$
\rho(f(a), f(b)) \leq \rho(a, b) \text { for all } a, b \in \mathbf{D} .
$$

In particular, holomorphic self maps of $\mathbf{D}$ do not increase the distance in the hyperbolic metric. Indeed

$$
\lambda_{\mathbf{D}}(f(z))\left|f^{\prime}(z)\right|=\lambda_{\mathbf{D}}(z) \text { for } z \in \mathbf{D},
$$

which follows easily by multiplying (2) by

$$
\frac{1}{|a-b|}=\left|\frac{1}{f(a)-f(b)} \cdot \frac{f(a)-f(b)}{a-b}\right|
$$

and take the limit as $a \rightarrow b$, and then finally set $b=z$. 
Observe that Pick's version of the Schwarz lemma, namely the inequality (2), guarantees that $\left|f^{*}(z)\right| \leq 1$ unless $f$ is a biholomorphic automorphism of the unit disk $\mathbf{D}$, and this means that we can measure the hyperbolic distance between two hyperbolic derivatives.

Before we state our main results, we need to fix up some notation. Let $\mathbf{D}(a, r)=$ $\{z \in \mathbf{C}:|z-a|<r\}$ denote the Euclidean open disk of radius $r$ centered at $a \in \mathbf{C}$. The closure of $\mathbf{D}(a, r)$ will be denoted by $\overline{\mathbf{D}}(a, r)$ and its boundary by $\partial \mathbf{D}(a, r)$. The open disk $\mathbf{D}(0, r)$ will denoted by $\mathbf{D}_{r}$, so that $\mathbf{D}:=\mathbf{D}_{1}$. For convenience, we use the notation $z=x+i y$ and $z=(x, y)$ interchangeably.

As for function spaces, $\mathcal{H}(G)$ is the linear space of holomorphic functions defined on the simply connected subset $G$ of $\mathbf{C}$. With the topology of local uniform convergence on compact subsets of $G$, the space $\mathcal{H}(G)$ becomes a topological space.

\section{Main results}

The first result is the extension of the Julia lemma on the unit disk endowed by the hyperbolic metric. Throughout the discussion, $f^{*}\left(z_{0}\right)$ denotes the hyperbolic derivative of $f$ at the point $z_{0}$ defined by (1), and the dot product $t \cdot \nabla$ with $t=$ $\left(t_{1}, t_{2}\right) \in \mathbf{R}^{2}$, is the usual operator notation

$$
t \cdot \nabla=t_{1} \frac{\partial}{\partial x}+t_{2} \frac{\partial}{\partial y} .
$$

Theorem 1. Let $r_{0} \in(0,1)$ and $z_{0} \in \partial \mathbf{D}_{r_{0}}$. Suppose that the function $f: \mathbf{D} \rightarrow$ $\mathbf{D}$ is holomorphic and $f(0)=0$. If

$$
\rho\left(0, f\left(z_{0}\right)\right)=\max _{|z| \leq r_{0}} \rho(0, f(z)),
$$

then there exists a real number $m$ with $|m| \geq 1$ and such that

$$
\frac{z_{0} f^{\prime}\left(z_{0}\right)}{f\left(z_{0}\right)}=m f^{*}\left(z_{0}\right) \frac{\left|z_{0}\right| \overline{f^{\prime}\left(z_{0}\right)}}{\left|f\left(z_{0}\right)\right|} \cdot \frac{\rho\left(0, f\left(z_{0}\right)\right)}{\rho\left(0, z_{0}\right)}
$$

and

$$
\begin{aligned}
& s^{2} B^{2}\left(z_{0}\right)\left[1-m\left(\frac{\rho\left(0, f\left(z_{0}\right)\right)}{\rho\left(0, z_{0}\right)}\right)^{2}\right] \\
& \leq \frac{1}{m} \rho\left(0, z_{0}\right)(t \cdot \nabla)^{2} \rho\left(0, z_{0}\right)-\rho\left(0, f\left(z_{0}\right)\right)(t \cdot \nabla)^{2} \rho\left(0, f\left(z_{0}\right)\right),
\end{aligned}
$$

for all $s \in \mathbf{R} \backslash\{0\}$ and $t=(s, s) \in \mathbf{R}^{2} \backslash\{(0,0)\}$, where

$$
B\left(z_{0}\right)=A\left(z_{0}\right)\left[\operatorname{Re}\left((1-i) f\left(z_{0}\right) \overline{f^{\prime}\left(z_{0}\right)}\right)\right],
$$

with

$$
A(z)=\frac{1}{|f(z)|\left(1-|f(z)|^{2}\right)} .
$$

Proof. We apply the method of Lagrange multipliers. Let $\epsilon>0$ be chosen to be sufficiently small so that the neighborhood of $\left(x_{0}, y_{0}\right)$ lies in $\mathbf{D}$, say $\Omega=$ $\left(x_{0}-\epsilon, x_{0}+\epsilon\right) \times\left(y_{0}-\epsilon, y_{0}+\epsilon\right) \subset \mathbf{D}$, and consider the real-valued function $F$ on $\Omega$ defined by

$$
F(x, y)=[\rho(0, f(z))]^{2}-\lambda\left\{[\rho(0, z)]^{2}-r_{0}^{2}\right\},
$$


where $\lambda \in \mathbf{R}$ and $z_{0}=x_{0}+i y_{0}$. For convenience, we let $f=u+i v$ and introduce

$$
\begin{aligned}
& h(x, y)=\rho(0, f(z))=\frac{1}{2} \log \left(\frac{1+\sqrt{u^{2}(x, y)+v^{2}(x, y)}}{1-\sqrt{u^{2}(x, y)+v^{2}(x, y)}}\right), \\
& g(x, y)=\rho(0, z)=\frac{1}{2} \log \left(\frac{1+\sqrt{x^{2}+y^{2}}}{1-\sqrt{x^{2}+y^{2}}}\right)
\end{aligned}
$$

so that

$$
F(x, y)=h^{2}(x, y)-\lambda g^{2}(x, y)
$$

By the assumption, $z_{0}$ is a point of local conditional maximum for the function $[\rho(0, f(z))]^{2}$ under the condition $\left[\rho\left(0, z_{0}\right)\right]^{2}=r_{0}^{2}$, and thus, by the first derivative test for local extremum, we obtain that

$$
F_{x}\left(z_{0}\right)=0=F_{y}\left(z_{0}\right)
$$

which is equivalent to $F_{x}\left(z_{0}\right)+i F_{y}\left(z_{0}\right)=0$, where $F_{x}$ and $F_{y}$ represent the partial derivatives of $F$ with respect to $x$ and $y$, respectively.

We need to find expression for the terms $h_{x}\left(z_{0}\right)+i h_{y}\left(z_{0}\right)$ and $g_{x}\left(z_{0}\right)+i g_{y}\left(z_{0}\right)$. By a direct computation, (7) gives that

$$
h_{x}(z)=\frac{1}{1-|f(z)|^{2}} \frac{\partial}{\partial x}(|f(z)|)=A(z)\left(u(z) u_{x}(z)+v(z) v_{x}(z)\right)
$$

and similarly

$$
h_{y}(z)=A(z)\left(u(z) u_{y}(z)+v(z) v_{y}(z)\right)
$$

where $A(z)$ is given by (6). If we take in these two equalities $u(x, y)=x$ and $v(x, y)=y$, it follows that $u_{x}(z)=v_{y}(z)=1$ and $u_{y}(z)=v_{x}(z)=0$. These observations show that

$$
g_{x}(z)=\frac{x}{|z|\left(1-|z|^{2}\right)} \quad \text { and } \quad g_{y}(z)=\frac{y}{|z|\left(1-|z|^{2}\right)} .
$$

Next, using (9), (10) and the Cauchy-Riemann equations $u_{x}\left(z_{0}\right)=v_{y}\left(z_{0}\right)$ and $u_{y}\left(z_{0}\right)=-v_{x}\left(z_{0}\right)$ (which hold since $f=u+i v$ is holomorphic), we compute

$$
h_{x}\left(z_{0}\right)+i h_{y}\left(z_{0}\right)=A\left(z_{0}\right) f\left(z_{0}\right)\left(u_{x}\left(z_{0}\right)-i v_{x}\left(z_{0}\right)\right)=\frac{f\left(z_{0}\right) \overline{f^{\prime}\left(z_{0}\right)}}{\left|f\left(z_{0}\right)\right|\left(1-\left|f\left(z_{0}\right)\right|^{2}\right)}
$$

by the definition of $A(z)$ and the fact that $f^{\prime}\left(z_{0}\right)=u_{x}\left(z_{0}\right)+i v_{x}\left(z_{0}\right)$. Since

$$
F_{x}\left(z_{0}\right)+i F_{y}\left(z_{0}\right)=2 h\left(z_{0}\right)\left[h_{x}\left(z_{0}\right)+i h_{y}\left(z_{0}\right)\right]-2 \lambda g\left(z_{0}\right)\left[g_{x}\left(z_{0}\right)+i g_{y}\left(z_{0}\right)\right],
$$

the condition $F_{x}\left(z_{0}\right)+i F_{y}\left(z_{0}\right)=0$ gives that

$$
h\left(z_{0}\right)\left[h_{x}\left(z_{0}\right)+i h_{y}\left(z_{0}\right)\right]=\lambda g\left(z_{0}\right)\left[g_{x}\left(z_{0}\right)+i g_{y}\left(z_{0}\right)\right]
$$

which is same as

$$
h\left(z_{0}\right)\left(\frac{f\left(z_{0}\right) \overline{f^{\prime}\left(z_{0}\right)}}{\left|f\left(z_{0}\right)\right|\left(1-\left|f\left(z_{0}\right)\right|^{2}\right)}\right)=\lambda g\left(z_{0}\right)\left(\frac{z_{0}}{\left|z_{0}\right|\left(1-\left|z_{0}\right|^{2}\right)}\right)
$$

and therefore,

$$
\frac{1-\left|z_{0}\right|^{2}}{1-\left|f\left(z_{0}\right)\right|^{2}}=\lambda \frac{z_{0}}{f\left(z_{0}\right)}\left|\frac{f\left(z_{0}\right)}{z_{0}}\right| \frac{1}{\overline{f^{\prime}\left(z_{0}\right)}} \frac{g\left(z_{0}\right)}{h\left(z_{0}\right)}
$$


Using this, we obtain that

$$
f^{*}\left(z_{0}\right)=\frac{1-\left|z_{0}\right|^{2}}{1-\left|f\left(z_{0}\right)\right|^{2}} \cdot f^{\prime}\left(z_{0}\right)=\lambda \frac{z_{0} f^{\prime}\left(z_{0}\right)}{f\left(z_{0}\right)} \cdot \frac{\left|f\left(z_{0}\right)\right|}{\left|z_{0}\right| f^{\prime}\left(z_{0}\right)} \cdot \frac{g\left(z_{0}\right)}{h\left(z_{0}\right)} .
$$

If in (13) we take $\lambda=1 / m$ (clearly $\lambda \neq 0$ ), we get the desired relation (3). Next, we claim that $|m| \geq 1$. From (13), it follows that

$$
\frac{h\left(z_{0}\right)}{g\left(z_{0}\right)}\left|f^{*}\left(z_{0}\right)\right|=\left|\frac{1}{m}\right| .
$$

But by Schwarz-Pick's lemma (see Lemma B) and Corollary C, we have that

$$
\left|f^{*}\left(z_{0}\right)\right| \leq 1 \text { and } h\left(z_{0}\right)=\rho\left(0, f\left(z_{0}\right)\right) \leq \rho\left(0, z_{0}\right)=g\left(z_{0}\right)
$$

(evidently we use the fact that $f(0)=0$ ). Therefore, we obtain that

$$
\left|\frac{1}{m}\right|=\frac{h\left(z_{0}\right)}{g\left(z_{0}\right)} \cdot\left|f^{*}\left(z_{0}\right)\right| \leq 1
$$

which proves that $|m| \geq 1$.

Because $z_{0}$ is a local maximum, the second differential of $F$ at the point $z_{0}$ is negative semidefinite. That is, the quadratic form $(t \cdot \nabla)^{2} F\left(z_{0}\right)$ for all $t=\left(t_{1}, t_{2}\right) \in$ $\mathbf{R}^{2} \backslash\{0\}$ is negative, where

$$
\begin{aligned}
(t \cdot \nabla)^{2} F\left(z_{0}\right) & =\left(\begin{array}{ll}
t_{1} & t_{2}
\end{array}\right)\left(\begin{array}{ll}
F_{x x}\left(z_{0}\right) & F_{x y}\left(z_{0}\right) \\
F_{y x}\left(z_{0}\right) & F_{y y}\left(z_{0}\right)
\end{array}\right)\left(\begin{array}{c}
t_{1} \\
t_{2}
\end{array}\right) \\
& =t_{1}^{2} F_{x x}\left(z_{0}\right)+2 t_{1} t_{2} F_{x y}\left(z_{0}\right)+t_{2}^{2} F_{y y} .
\end{aligned}
$$

We now set $t_{1}=t_{2}=s \in \mathbf{R} \backslash\{0\}$. Then it is a simple exercise to see that

$$
\begin{aligned}
(t \cdot \nabla)^{2} F\left(z_{0}\right)= & 2 s^{2}\left[\left(h_{x}\left(z_{0}\right)+h_{y}\left(z_{0}\right)\right)^{2}-\frac{1}{m}\left(g_{x}\left(z_{0}\right)+g_{y}\left(z_{0}\right)\right)^{2}\right] \\
& +2 h\left(z_{0}\right)(t \cdot \nabla)^{2} h\left(z_{0}\right)-2 \frac{1}{m} g\left(z_{0}\right)(t \cdot \nabla)^{2} g\left(z_{0}\right),
\end{aligned}
$$

where $t=(s, s) \in \mathbf{R}^{2} \backslash\{(0,0)\}$. Equation (12) (with $\lambda=1 / m$ ) yields that

$$
\frac{g_{x}\left(z_{0}\right)+g_{y}\left(z_{0}\right)}{h_{x}\left(z_{0}\right)+h_{y}\left(z_{0}\right)}=m \cdot \frac{h\left(z_{0}\right)}{g\left(z_{0}\right)}=m \cdot \frac{\rho\left(0, f\left(z_{0}\right)\right)}{\rho\left(0, z_{0}\right)} \text {. }
$$

Moreover, (11) quickly implies that

$$
\begin{aligned}
h_{x}\left(z_{0}\right)+h_{y}\left(z_{0}\right) & =A\left(z_{0}\right)\left[\operatorname{Re}\left(f\left(z_{0}\right) \overline{f^{\prime}\left(z_{0}\right)}\right)+\operatorname{Im}\left(f\left(z_{0}\right) \overline{f^{\prime}\left(z_{0}\right)}\right)\right] \\
& =A\left(z_{0}\right) \operatorname{Re}\left((1-i) f\left(z_{0}\right) \overline{f^{\prime}\left(z_{0}\right)}\right)=B\left(z_{0}\right) .
\end{aligned}
$$

Finally, because $(t \cdot \nabla)^{2} F\left(z_{0}\right) \leq 0$, the last two relations give the desired inequality (4). The proof is complete.

We now introduce a class of holomorphic functions for which Theorem 1 is applicable. Let $\mathcal{Q}$ denote the class of functions $q$ holomorphic and one-to-one on $\overline{\mathbf{D}} \backslash E(q)$, where $E(q)=\{\zeta \in \partial \mathbf{D}: q(z) \rightarrow \infty$ as $z \rightarrow \zeta\}$. Note that on $q\left(\zeta_{0}\right) \neq 0$ on $\overline{\mathbf{D}} \backslash E(q)$.

Theorem 2. Let $p, q \in \mathcal{H}(\mathbf{D})$ with $p(0)=q(0)$, where $q(k z) \in \mathcal{Q}$. Suppose that there exist an $z_{0}=x_{0}+i y_{0} \in \mathbf{D}$ and $\zeta_{0} \in \partial \mathbf{D} \backslash E(q)$ such that $p\left(z_{0}\right)=q\left(k \zeta_{0}\right)$ and 
$p\left(\mathbf{D}_{r_{0}}\right) \subset q\left(\mathbf{D}_{k}\right)$ for some $k \in(0,1)$, where $r_{0}=\left|z_{0}\right|$ and $q$ is locally univalent in $\overline{\mathbf{D}_{k}}$. Then there exists an $m \in \mathbf{R}$ with $|m| \geq 1$ such that

$$
\frac{z_{0} p^{\prime}\left(z_{0}\right)}{\left|z_{0}\right|\left|p^{\prime}\left(z_{0}\right)\right|^{2}} \cdot \frac{\rho\left(0, r_{0}\right)}{1-\left|z_{0}\right|^{2}}=m \cdot \frac{\zeta_{0} q^{\prime}\left(k \zeta_{0}\right)}{\left|q^{\prime}\left(k \zeta_{0}\right)\right|^{2}} \cdot \frac{\rho(0, k)}{1-k^{2}}
$$

and

$$
\begin{aligned}
& B^{2}\left(z_{0}\right)\left[\frac{3 k^{2}+k-1}{k}-m\left(\frac{\rho(0, k)}{\rho\left(0, r_{0}\right)}\right)^{2}\right] \\
& \leq \frac{\rho\left(0, r_{0}\right)}{m} H\left(z_{0}\right)-\left[\left|\frac{p^{\prime}\left(z_{0}\right)}{k q^{\prime}\left(k \zeta_{0}\right)}\right|^{2}-K\left(z_{0}\right)\right] \frac{2 \rho(0, k)}{1-k^{2}}
\end{aligned}
$$

where

$$
\begin{aligned}
& B\left(z_{0}\right)=\frac{1}{1-k^{2}}\left[\operatorname{Re} \frac{\overline{p^{\prime}\left(z_{0}\right)}}{\overline{\zeta_{0} q^{\prime}\left(k \zeta_{0}\right)}}+\operatorname{Im} \frac{\overline{p^{\prime}\left(z_{0}\right)}}{\overline{\zeta_{0} q^{\prime}\left(k \zeta_{0}\right)}}\right], \\
& H\left(z_{0}\right)=\frac{1}{\left|z_{0}\right|\left(1-\left|z_{0}\right|^{2}\right)}\left[\frac{\left(x_{0}+y_{0}\right)^{2}\left(3\left|z_{0}\right|^{2}-1\right)}{\left|z_{0}\right|^{2}\left(1-\left|z_{0}\right|^{2}\right)}+2\right], \\
& K\left(z_{0}\right)=\operatorname{Im}\left[\overline{\zeta_{0}} \frac{p^{\prime \prime}\left(z_{0}\right)\left[q^{\prime}\left(k \zeta_{0}\right)\right]^{2}-q^{\prime \prime}\left(k \zeta_{0}\right)\left[p^{\prime}\left(z_{0}\right)\right]^{2}}{\left[q^{\prime}\left(k \zeta_{0}\right)\right]^{3}}\right] .
\end{aligned}
$$

Proof. By the assumption we have that $p\left(\overline{\mathbf{D}}_{r_{0}}\right) \subset q\left(\overline{\mathbf{D}}_{k}\right)$. Observe that $p\left(\overline{\mathbf{D}}_{r_{0}}\right)$ is bounded because $p$ is holomorphic in $\mathbf{D}$. Define $f: \overline{\mathbf{D}}_{r_{0}} \rightarrow \Omega \subset \mathbf{D}$ by

$$
f(z)=q^{-1}(p(z)), \quad z \in \overline{\mathbf{D}}_{r_{0}} .
$$

Then $f \in \mathcal{H}\left(\overline{\mathbf{D}}_{r_{0}}\right), f(0)=q^{-1}(q(0))=0$, and $|f(z)| \leq k=\left|f\left(z_{0}\right)\right|$ for $|z| \leq r_{0}$, where $f\left(z_{0}\right)=q^{-1}\left(p\left(z_{0}\right)\right)=q^{-1}\left(q\left(k \zeta_{0}\right)\right)=k \zeta_{0} \in \partial \mathbf{D}$. Since $p(z)=q(f(z))$, we see that $z p^{\prime}(z)=z q^{\prime}(f(z)) \cdot f^{\prime}(z)$ and thus, at the point $z_{0}$, we rewrite

$$
z_{0} p^{\prime}\left(z_{0}\right)=z_{0} q^{\prime}\left(f\left(z_{0}\right)\right) f^{\prime}\left(z_{0}\right)=\left(\frac{z_{0} f^{\prime}\left(z_{0}\right)}{f\left(z_{0}\right)}\right) k \zeta_{0} q^{\prime}\left(k \zeta_{0}\right) \quad \text { and } \quad f^{\prime}\left(z_{0}\right)=\frac{p^{\prime}\left(z_{0}\right)}{q^{\prime}\left(k \zeta_{0}\right)}
$$

Observe that $\rho\left(0, f\left(z_{0}\right)\right)=\rho\left(0, k \zeta_{0}\right)=\rho(0, k)$ and by the last condition one has $\left|f^{\prime}\left(z_{0}\right)\right|=\left|p^{\prime}\left(z_{0}\right) / q^{\prime}\left(k \zeta_{0}\right)\right|$. These observations and (16) imply that the representation (3) in Theorem 1 is easily seen to be equivalent to (14).

For the proof of (15), we need to find an equivalent expression for the inequality (4) in Theorem 1. To do this, we need first to calculate

$$
(t \cdot \nabla)^{2} h(z)=s^{2}\left[h_{x x}(z)+2 h_{x y}(z)+h_{y y}(z)\right]
$$

at $z=z_{0}$, for $t=(s, s) \in \mathbf{R}^{2} \backslash\{(0,0)\}$ with $h(z)=\rho(0, f(z))$. By (9), one easily obtains that

$$
\begin{aligned}
& h_{x x}=A_{x}\left(u u_{x}+v v_{x}\right)+A\left(u u_{x x}+v v_{x x}+u_{x}^{2}+v_{x}^{2}\right), \\
& h_{y y}=A_{y}\left(u u_{y}+v v_{x}\right)+A\left(u u_{y y}+v v_{y y}+u_{y}^{2}+v_{y}^{2}\right), \\
& h_{x y}=A_{y}\left(u u_{x}+v v_{x}\right)+A\left(u_{y} u_{x}+u u_{x y}+v_{y} v_{x}+v v_{x y}\right),
\end{aligned}
$$

where $f=u+i v$ and $A$ is given by (6). Moreover,

$$
A_{x}=\frac{\left(3|f|^{2}-1\right)\left(u u_{x}+v v_{x}\right)}{|f|^{3}\left(1-|f|^{2}\right)^{2}} \quad \text { and } \quad A_{y}=\frac{\left(3|f|^{2}-1\right)\left(u u_{y}+v v_{y}\right)}{|f|^{3}\left(1-|f|^{2}\right)^{2}} .
$$


Using these, a computation gives that

$$
\begin{aligned}
(t \cdot \nabla)^{2} h= & \frac{s^{2}\left(3|f|^{2}-1\right)}{|f|^{3}\left(1-|f|^{2}\right)^{2}}\left[\left(u u_{x}+v v_{x}\right)^{2}+2\left(u u_{x}+v v_{x}\right)\left(u u_{y}+v v_{y}\right)+\left(u u_{y}+v v_{y}\right)^{2}\right] \\
& +2 s^{2} A\left[\left|f^{\prime}\right|^{2}+u u_{x y}+v v_{x y}\right] .
\end{aligned}
$$

Since $2\left(u u_{x y}+v v_{x y}\right)=u(t \cdot \nabla)^{2} u+v(t \cdot \nabla)^{2} v=2 \operatorname{Im}\left(f \overline{f^{\prime \prime}}\right)$, the last relation at $z=z_{0}$ shows that

$$
(t \cdot \nabla)^{2} h\left(z_{0}\right)=s^{2}\left\{\frac{\left(3\left|f\left(z_{0}\right)\right|^{2}-1\right)}{\left|f\left(z_{0}\right)\right|} B^{2}\left(z_{0}\right)+2 A\left|f^{\prime}\left(z_{0}\right)\right|^{2}+2 A\left(z_{0}\right) \operatorname{Im}\left(f\left(z_{0}\right) \overline{f^{\prime \prime}\left(z_{0}\right)}\right)\right\},
$$

where

$$
C\left(z_{0}\right)=u\left(z_{0}\right)\left(u_{x}\left(z_{0}\right)+u_{y}\left(z_{0}\right)\right)+v\left(z_{0}\right)\left(v_{x}\left(z_{0}\right)+v_{y}\left(z_{0}\right)\right)=\operatorname{Re}\left((1-i) f\left(z_{0}\right) \overline{f^{\prime}\left(z_{0}\right)}\right)
$$

and $B\left(z_{0}\right)=A\left(z_{0}\right) C\left(z_{0}\right)$ is given by (5) so that

$$
B\left(z_{0}\right)=\frac{1}{1-k^{2}} \operatorname{Re}\left((1-i) \zeta_{0} \frac{\overline{p^{\prime}\left(z_{0}\right)}}{\overline{q^{\prime}\left(k \zeta_{0}\right)}}\right)=\frac{1}{1-k^{2}} \operatorname{Re}\left((1-i) \frac{\overline{p^{\prime}\left(z_{0}\right)}}{\overline{\zeta_{0} q^{\prime}\left(k \zeta_{0}\right)}}\right),
$$

since $\left|\zeta_{0}\right|=1$.

If we take in the above expression $u(x, y)=x$ and $v(x, y)=y$, then from (17) we find after computation that

$$
(t \cdot \nabla)^{2} g\left(z_{0}\right)=\frac{1}{\left|z_{0}\right|\left(1-\left|z_{0}\right|^{2}\right)}\left[\frac{\left(x_{0}+y_{0}\right)^{2}\left(3\left|z_{0}\right|^{2}-1\right)}{\left|z_{0}\right|^{2}\left(1-\left|z_{0}\right|^{2}\right)}+2\right] s^{2}=H\left(z_{0}\right) s^{2} .
$$

The second relation (4) in Theorem 1 becomes

$$
\begin{aligned}
& s^{2} B^{2}\left(z_{0}\right)\left[\frac{3\left|f\left(z_{0}\right)\right|^{2}+\left|f\left(z_{0}\right)\right|-1}{\left|f\left(z_{0}\right)\right|}-m\left(\frac{\rho\left(0, f\left(z_{0}\right)\right.}{\rho\left(0, r_{0}\right)}\right)^{2}\right] \\
& \leq \frac{1}{m} \rho\left(0, r_{0}\right)(t \cdot \nabla)^{2} g\left(z_{0}\right)-2 A\left(z_{0}\right) s^{2}\left[\left|f^{\prime}\left(z_{0}\right)\right|^{2}-\operatorname{Im}\left(\overline{f\left(z_{0}\right)} f^{\prime \prime}\left(z_{0}\right)\right)\right] .
\end{aligned}
$$

Since $p(z)=q(f(z))$, we have $p^{\prime \prime}\left(z_{0}\right)=q^{\prime \prime}\left(k \zeta_{0}\right)\left(f^{\prime}\left(z_{0}\right)\right)^{2}+q^{\prime}\left(k \zeta_{0}\right) f^{\prime \prime}\left(z_{0}\right)$ and thus, using the second relation in (16) it follows easily that

$$
f^{\prime \prime}\left(z_{0}\right)=\frac{p^{\prime \prime}\left(z_{0}\right)\left[q^{\prime}\left(k \zeta_{0}\right)\right]^{2}-q^{\prime \prime}\left(k \zeta_{0}\right)\left[p^{\prime}\left(z_{0}\right)\right]^{2}}{\left[q^{\prime}\left(k \zeta_{0}\right)\right]^{3}}
$$

and the desired conclusion follows by substituting this, $f\left(z_{0}\right)=k \zeta_{0}$, and using (16) in the last expression. The proof is complete.

We need to introduce the following definition.

Definition 1. Let $f, g \in \mathcal{H}(\mathbf{D})$. We say that the function $f$ is subordinate to $g$, written $f \prec g$ or $f(z) \prec g(z)$, if there exists a function $w$ holomorphic in $\mathbf{D}$, with $w(0)=0, \rho(0, w(z))<\operatorname{arctanh} k$ for some $k \in(0,1)$ and such that $f(z)=g(w(z))$.

Note that $\rho(0, w(z))<\operatorname{arctanh} k$ is equivalent to $|w(z)|<k$ in $\mathbf{D}$, and thus, $k \rightarrow 1^{-}$gives us the standard definition of subordination. We also note that if $g$ is univalent, then it is easy to show that $f \prec g$ if and only if $f(0)=g(0)$ and $f(\mathbf{D}) \subset g(k \mathbf{D})$ for some $k \in(0,1)$. 
Corollary 1. Suppose that $p, q \in \mathcal{H}(\mathbf{D})$ with $p(0)=q(0)$, where $q(k z) \in \mathcal{Q}$. If $p$ is not subordinate to $q$, then there exist an $z_{0} \in \mathbf{D}$ with $\left|z_{0}\right|=r_{0}, k \in(0,1)$, $\zeta_{0} \in \partial \mathbf{D} \backslash E(q)$, a real number $m$ with $|m| \geq 1$ and such that $p\left(\mathbf{D}_{r_{0}}\right) \subset q\left(\mathbf{D}_{k}\right)$,

$$
p\left(z_{0}\right)=q\left(k \zeta_{0}\right)
$$

and satisfies the conditions (14) and (15).

Proof. Suppose that $p$ and $q$ are holomorphic in $\mathbf{D}$ such that $p(z) \nprec q(z)$. In view of Schwarz's lemma, we obtain that $p(\mathbf{D}) \nsubseteq q(k \mathbf{D})=q\left(\mathbf{D}_{k}\right)$. Since $p$ and $q$ are holomorphic in $\mathbf{D}$, we can define

$$
r_{0}=\sup \left\{r: p\left(\mathbf{D}_{r}\right) \subset q\left(\mathbf{D}_{k}\right)\right\} .
$$

The fact that $p(\mathbf{D}) \nsubseteq q\left(\mathbf{D}_{k}\right)$ implies that there exists an $r_{0} \in(0,1)$ for which $p\left(\mathbf{D}_{r_{0}}\right) \subset$ $q\left(\mathbf{D}_{k}\right)$ and $p\left(\overline{\mathbf{D}}_{r_{0}}\right) \nsubseteq q\left(\mathbf{D}_{k}\right)$. But $p\left(\overline{\mathbf{D}}_{r_{0}}\right) \subset \overline{q\left(\mathbf{D}_{k}\right)}=\partial q\left(\mathbf{D}_{k}\right)$, and hence, there exists an $z_{0} \in \partial \mathbf{D}_{r_{0}}$ such that $p\left(z_{0}\right) \in \partial q\left(\mathbf{D}_{k}\right)$. This provides the existence of $\zeta_{0} \in \partial \mathbf{D}$ such that $p\left(z_{0}\right)=q\left(k \zeta_{0}\right)$. Now we apply Theorem 2 and obtain the desired conclusions of the corollary.

\section{Particular case}

We consider the case where $q(\mathbf{D})$ is a disk. Let

$$
w=q(z)=M \frac{M z+a}{M+\bar{a} z},
$$

with $M>0$ and $|a|<M$. Then $q(\mathbf{D})=\mathbf{D}_{M}, q(0)=a$, the function $q$ is holomorphic and univalent for $|z|<M /|a|$ and, in particular, in the closed unit disk $\overline{\mathbf{D}}$. Moreover, an easy computation shows that

$$
z=q^{-1}(w)=\frac{M(w-a)}{M^{2}-\bar{a} w}, q^{\prime}(z)=\frac{M\left(M^{2}-|a|^{2}\right)}{(M+\bar{a} z)^{2}} \text { and } q^{\prime \prime}(z)=-\frac{2 M\left(M^{2}-|a|^{2}\right) \bar{a}}{(M+\bar{a} z)^{3}} .
$$

If there exist points $z_{0} \in \mathbf{D}$ and $\zeta_{0} \in \partial \mathbf{D}$ such that $p\left(z_{0}\right)=q\left(k \zeta_{0}\right)$ for some $k \in(0,1)$ and $\rho(0, p(z))<M$ for all $z$ such that $|z|<\left|z_{0}\right|$, then $\rho\left(0, p\left(z_{0}\right)\right)=\rho\left(0, q\left(k \zeta_{0}\right)\right)=M$. For convenience, we denote $M\left[p\left(z_{0}\right)-a\right]=a_{1}, M^{2}-\bar{a} p\left(z_{0}\right)=a_{2}$ and $M^{2}-|a|^{2}=a_{3}$. Thus, we have

$k \zeta_{0}=q^{-1}\left(p\left(z_{0}\right)\right)=\frac{M\left[p\left(z_{0}\right)-a\right]}{M^{2}-\bar{a} p\left(z_{0}\right)}$ and $k \zeta_{0} q^{\prime}\left(k \zeta_{0}\right)=\frac{\left[M^{2}-\bar{a} p\left(z_{0}\right)\right]\left[p\left(z_{0}\right)-a\right]}{M^{2}-|a|^{2}}=\frac{a_{1} a_{2}}{M a_{3}}$

so that

$$
k\left|q^{\prime}\left(k \zeta_{0}\right)\right|=\frac{\left|a_{1} a_{2}\right|}{M a_{3}}, \quad \frac{\zeta_{0} q^{\prime}\left(k \zeta_{0}\right)}{\left|q^{\prime}\left(k \zeta_{0}\right)\right|^{2}}=\frac{M k a_{1} a_{2} a_{3}}{\left|a_{1} a_{2}\right|^{2}}, \quad \text { and } \quad q^{\prime \prime}\left(\zeta_{0}\right)=-\frac{2 \bar{a} a_{2}^{3}}{\left(a_{3} M\right)^{2}} .
$$

Finally, we compute

$$
\begin{aligned}
\overline{\zeta_{0}} \frac{p^{\prime \prime}\left(z_{0}\right)\left[q^{\prime}\left(k \zeta_{0}\right)\right]^{2}-q^{\prime \prime}\left(k \zeta_{0}\right)\left[p^{\prime}\left(z_{0}\right)\right]^{2}}{\left[q^{\prime}\left(k \zeta_{0}\right)\right]^{3}} & =k \frac{p^{\prime \prime}\left(z_{0}\right)\left[k \zeta_{0} q^{\prime}\left(k \zeta_{0}\right)\right]^{2}-k^{2} \zeta_{0}^{2} q^{\prime \prime}\left(k \zeta_{0}\right)\left[p^{\prime}\left(z_{0}\right)\right]^{2}}{\left[k \zeta_{0} q^{\prime}\left(k \zeta_{0}\right)\right]^{3}} \\
& =\frac{a_{3} M k}{a_{1} a_{2}^{2}}\left[a_{2} p^{\prime \prime}\left(z_{0}\right)+2 \bar{a}\left(p^{\prime}\left(z_{0}\right)\right)^{2}\right] .
\end{aligned}
$$

Using these relations with Theorem 2, we obtain the following result. 
Theorem 3. Let $p \in \mathcal{H}(\mathbf{D})$, with $p(0)=a$ and let $z_{0} \in \mathbf{D}$ such that

$$
\rho\left(0, p\left(z_{0}\right)\right)=\max \left\{\rho(0, p(z)):|z| \leq\left|z_{0}\right|\right\} .
$$

Suppose that $M>0,|a|<M$ and $k$ is such that $k=\left|M\left[p\left(z_{0}\right)-a\right] /\left[M^{2}-\bar{a} p\left(z_{0}\right)\right]\right|$. Then exists a number $m$ such that $|m| \geq 1$ with

$$
\frac{z_{0} p^{\prime}\left(z_{0}\right)}{\left|z_{0}\right|\left|p^{\prime}\left(z_{0}\right)\right|^{2}} \cdot \frac{\rho\left(0, r_{0}\right)}{1-\left|z_{0}\right|^{2}}=m \cdot \frac{M k a_{1} a_{2} a_{3}}{\left|a_{1} a_{2}\right|^{2}} \cdot \frac{\rho(0, k)}{1-k^{2}}
$$

and

$$
\begin{aligned}
& B^{2}\left(z_{0}\right)\left[\frac{3 k^{2}+k-1}{k}-m\left(\frac{\rho(0, k)}{\rho\left(0, r_{0}\right)}\right)^{2}\right] \\
& \leq \frac{\rho\left(0, r_{0}\right)}{m} H\left(z_{0}\right)-\left[\frac{\left|p^{\prime}\left(z_{0}\right)\right|^{2} M^{2} a_{3}^{2}}{\left|a_{1} a_{2}\right|^{2}}-K\left(z_{0}\right)\right] \frac{2 \rho(0, k)}{1-k^{2}}
\end{aligned}
$$

where

$$
\begin{aligned}
& B\left(z_{0}\right)=\frac{M k a_{3}}{1-k^{2}}\left[\operatorname{Re} \frac{\overline{p^{\prime}\left(z_{0}\right)}}{\overline{a_{1} a_{2}}}+\operatorname{Im} \frac{\overline{p^{\prime}\left(z_{0}\right)}}{\overline{a_{1} a_{2}}}\right], \\
& H\left(z_{0}\right)=\frac{1}{\left|z_{0}\right|\left(1-\left|z_{0}\right|^{2}\right)}\left[\frac{\left(x_{0}+y_{0}\right)^{2}\left(3\left|z_{0}\right|^{2}-1\right)}{\left|z_{0}\right|^{2}\left(1-\left|z_{0}\right|^{2}\right)}+2\right], \\
& K\left(z_{0}\right)=\operatorname{Im}\left[\frac{a_{3} M k}{a_{1} a_{2}^{2}}\left[a_{2} p^{\prime \prime}\left(z_{0}\right)+2 \bar{a}\left(p^{\prime}\left(z_{0}\right)\right)^{2}\right]\right] .
\end{aligned}
$$

We remark that if we choose $a=0$ and $p(\mathbf{D})=\mathbf{D}$ in Theorem 3 , then $k$ could be clearly taken as $\left|p\left(z_{0}\right)\right|$ and hence, in this choice (19) is same as (3) and hence, Theorem 3 reduces to Theorem 1 .

\section{References}

[Be] Beardon, A. F.: The Schwarz-Pick lemma for derivatives. - Proc. Amer. Math. Soc. 125, 1997, 3255-3256.

[BC] Bernal-González, L., and M. C. Calderón-Moreno: Two hyperbolic Schwarz lemmas. - Bull. Austral. Math. Soc. 66, 2002, 17-24.

[Bo] BoAs, H. P.: Julius and Julia: mastering the art of the Schwarz lemma. - Amer. Math. Monthly 117, 2010, 770-785.

[Cl] Clunie, J. G.: Some remarks on extreme points in function theory. - In: Aspects of Contemporary Complex Analysis, Proc. NATO Adv. Study Inst., University of Durham, Durham, UK, 1979, Academic Press, London, 1980, 137-146.

[CV1] CurT, P., and Cs. VArga: Jack's, Miller's and Mocanu's lemma for holomorphic mappings defined on domains with differentiable boundary of class $\mathbf{C}^{2}$. - Studia Univ. Babes-Bolyai Mathematica 40:2, 1995, 41-52.

[CV2] Curt, P., and Cs. Varga: Jack, Miller and Mocanu lemma for holomorphic mappings in $\mathbf{C}^{n}$. - Stud. Cercet. Mat. 49:1-2, 1997, 39-45.

[IT] Imayoshi, Y., and M. TAniguchi: An introduction to Teichmüller spaces. - SpringerVerlag, Tokyo, 1992.

[Ja] JACK, I. S.: Functions starlike and convex of order $\alpha$. - J. London Math. Soc. 3:2, 1971, 469-474. 
[KP] Kohr, G., and C. PIntea: An extension of the Jack's-Miller's-Mocanu's lemma for holomorphic mappings defined on some domains in $\mathbf{C}^{n}$. - Libertas Math. 16, 1996, 61-71.

[MM1] Miller, S. S., and P. T. Mocanu: Second order differential inequalities in the complex plane. - J. Math. Anal. Appl. 65, 1978, 289-305.

[MM2] Miller, S. S., and P. T. Mocanu: Differential subordinations and univalent functions. Michigan Math. J. 28, 1981, 157-171.

[MM3] Miller, S. S., and P. T. Mocanu: Differential subordinations. Theory and applications. - Marcel Dekker Inc., New York-Basel, 2000.

[Os] Osserman, R.: A new variant of the Schwarz-Pick-Ahlfors lemma. - Manuscripta Math. 100:2, 1999, 123-129.

Received 15 September 2014 • Accepted 10 April 2015 